\title{
COMBINATORIAL INTERPRETATIONS OF RAMANUJAN'S TAU FUNCTION
}

\author{
FRANK GARVAN AND MICHAEL J. SCHLOSSER
}

\begin{abstract}
We use a $q$-series identity by Ramanujan to give a combinatorial interpretation of Ramanujan's tau function which involves $t$-cores and a new class of partitions which we call $(m, k)$-capsids. The same method can be applied in conjunction with other related identities yielding alternative combinatorial interpretations of the tau function.
\end{abstract}

\section{INTRODUCTION}

Ramanujan's [24] tau function $\tau(n)$ is defined to be the coefficient of $q^{n}$ in the 24th power of Dedekind's eta function $\eta(q):=q^{\frac{1}{24}}(q ; q)_{\infty}$, i.e.,

$$
\sum_{n \geq 1} \tau(n) q^{n}=q(q ; q)_{\infty}^{24}
$$

Here we are using standard $q$-series notation (cf. [14])

$$
(a ; q)_{n}:=\prod_{j=0}^{n-1}\left(1-a q^{j}\right),
$$

where $a \in \mathbb{C}, 0<|q|<1$ and $n$ is an nonnegative integer (for $n=0$ the expression $(a ; q)_{0}$ reduces to an empty product which is defined to be 1) or infinity. For brevity, we also frequently use the compact notation

$$
\left(a_{1}, \ldots, a_{m} ; q\right)_{n}:=\left(a_{1} ; q\right)_{n} \ldots\left(a_{m} ; q\right)_{n} .
$$

The tau function possesses very nice arithmetic properties, see [26]. In particular, $\tau(n)$ is a multiplicative function, as originally observed by Ramanujan and later proved by Mordell [23]. Lehmer [19] conjectured that the tau function never vanishes, which is still open. The tau function oscillates and assumes positive and negative integer values. Dyson [7, Eq.(2), p.636] revealed a nice formula for $\tau(n)$ which is actually a special case of one of the Macdonald identities [20]. Although the latter formula does have a strong combinatorial flavour, as it involves an alternating multiple sum of rational numbers the

2010 Mathematics Subject Classification. Primary 05A15; Secondary 05A17, 05A30, 11F11, 11P81, 11P83, 11P84.

Key words and phrases. Ramanujan's tau function, $q$-series, partitions, $t$-cores, $(m, k)$-capsids.

Partly supported by a grant from the Simon's Foundation (\#318714).

Partly supported by FWF Austrian Science Fund grant F50-08 within the SFB "Algorithmic and enumerative combinatorics". 
formula is not easily seen to be related to a direct counting of special combinatorial objects.

We are interested in finding combinatorial interpretations for $\tau(n)$. We succeed by employing $q$-series identities of a specific type, together with the use of generating functions for $t$-cores and for a new class of partitions which we call $(m, k)$-capsids. The main idea is to split the generating function of $\tau(n)$ using an identity of the type (3.9) in a nonnegative component and a nonpositive component, see (4.1), where all components admit easy combinatorial interpretations in terms of $t$-cores and $(m, k)$-capsids.

\section{Partitions, $t$-CORES, And $(m, k)$-CAPSids}

Let $\mathbb{N}$ denote the set of positive integers. A partition $\lambda$ of a positive integer $n$ (cf. [2]) is a nonincreasing sequence of positive integers (called the parts), $\lambda=\left(\lambda_{1}, \lambda_{2}, \ldots, \lambda_{l}\right)$, such that $\lambda_{1}+\lambda_{2}+\cdots+\lambda_{l}=n$. If $\lambda$ is a partition of $n$, then we write $\lambda \vdash n$ or $|\lambda|=n$. We can identitfy a partition $\lambda=\left(\lambda_{1}, \lambda_{2}, \ldots, \lambda_{l}\right)$ with its Ferrers diagram, denoted by $[\lambda]$, defined to be the set of cells $\left\{(i, j) \in \mathbb{N}^{2}: 1 \leq i \leq l, 1 \leq j \leq \lambda_{i}\right\}$. For a cell $(i, j) \in[\lambda]$, the hook $H_{\lambda}(i, j)$ of $\lambda$ is the following subset of the Ferrers diagram $[\lambda]$ : $H_{\lambda}(i, j)=\{(i, t) \in[\lambda]: t \geq j\} \cup\{(s, j) \in[\lambda]: s>i\}$. The cardinality of the hook $H_{\lambda}(i, j)$ is the hooklength $h_{\lambda}(i, j)$.

Sometimes it is convenient to write a partition $\lambda$ not as a nonincreasing sequence of positive integers but rather as a increasing sequence of integers with finitely many nonzero multiplicities, formally written in the form $\lambda=\left(1^{\mu_{1}}, 2^{\mu_{2}}, \ldots\right)$. Here, each positive integer $j \in \mathbb{N}$ has an nonnegative multiplicity $\mu_{j}$, correpsonding to the number of times the part $j$ occurs in $\lambda$. If $\lambda \vdash n$, we must have $\sum_{j \geq 1} j \mu_{j}=n$.

For our combinatorial interpretations of Ramanujan's tau function we will use two special classes of partitions: $t$-cores and $(m, k)$-capsids ${ }^{1}$, the latter are introduced here for the first time.

$t$-Cores have been introduced in [16, Sec. 2.7] where they have been shown to be useful for the recursive evaluation of the irreducible charcters of the symmetric group. A partition $\lambda$ is a $t$-core if, and only if, $\lambda$ contains no hooks whose lengths are multiples of $t$. Let $c(t, n)$ denote the number of $t$-core partitions of $n$. The generating function for $t$-cores was computed in [12]:

$$
T_{t}(q)=1+\sum_{n=1}^{\infty} c(t, n) q^{n}=\frac{\left(q^{t} ; q^{t}\right)_{\infty}^{t}}{(q ; q)_{\infty}} .
$$

See also [16, Theorem 2.7.17, p.80]. $t$-Cores have attracted broad interest and were studied from various points of view, in particular, with focus on some of their combinatorial [10] or analytic [1, 15] properties.

\footnotetext{
${ }^{1}$ In medical microbiology, the core is the inner structure of a viral particle (which consists of structural proteins and genom), such as a rotavirus; it is surrounded by an (inner and an outer) capsid, the protein shell of a virus.
} 
Let $m, k \in \mathbb{N}, m \geq 2$. For $0<k<m$ we say that a partition $\pi$ is an $(m, k)$-capsid if, and only if, the possible parts of $\pi$ are $m-k$ or are congruent to 0 or $k \bmod m$, and satisfy the following two conditions:

(i) if $\mu_{m-k}=0$, i.e., $m-k$ is not a part, then all parts are congruent to $k \bmod m$

(ii) if $\mu_{m-k}>0$, then $m-k$ is the smallest part and the largest part congruent to 0 mod $m$ is $\leq m \cdot \mu_{m-k}$ and all parts congruent to $k \bmod m$ (different from $m-k$, if $k=m / 2$ ) are $>m \cdot \mu_{m-k}$.

Let $\gamma(m, k, n)$ be the number of $(m, k)$-capsid partitions of $n$. Then, from the combinatorial definition it is clear that we have the following generating function

$$
C_{m, k}(q)=1+\sum_{n=1}^{\infty} \gamma(m, k, n) q^{n}=\sum_{n=0}^{\infty} \frac{q^{(m-k) n}}{\left(q^{m} ; q^{m}\right)_{n}\left(q^{m n+k} ; q^{m}\right)_{\infty}} .
$$

Example 1. Let $m=5$ and $k=1$. There are seven $(5,1)$-capsid partitions of 16 :

$$
\left(1^{16}\right), \quad\left(4^{4}\right), \quad\left(1^{10}, 6\right), \quad\left(1^{4}, 6^{2}\right), \quad\left(4,6^{2}\right), \quad\left(1^{5}, 11\right), \quad(16)
$$

so that $a(5,1,16)=7$.

It turns out the generating function $C_{m, k}(q)$ is also an infinite product. By [4, Eq. (4.2)] we have

$$
\frac{(a t ; q)_{\infty}}{(a ; q)_{\infty}(t ; q)_{\infty}}=\sum_{n=0}^{\infty} \frac{t^{n}}{(q)_{n}\left(a q^{n} ; q\right)_{\infty}}
$$

which is a generalization of [9, Eq. (5.7)]. This identity also follows immediately from the $q$-binomial theorem [14, Eq. (II.3)]. Now the $(q, a, t) \mapsto\left(q^{m}, q^{k}, q^{m-k}\right)$ case of 2.2 , together with 2.1), immediately establishes the following result:

Proposition 1. The generating function for $(m, k)$-capsids is

$$
C_{m, k}(q)=\frac{\left(q^{m} ; q^{m}\right)_{\infty}}{\left(q^{k} ; q^{m}\right)_{\infty}\left(q^{m-k} ; q^{m}\right)_{\infty}}
$$

Since $C_{m, k}(q)=C_{m, m-k}(q)$, by inspection, we immediately deduce that the numbers of $(m, k)$ - and $(m, m-k)$-capsid partitions of $n$ are equinumerous:

$$
\gamma(m, k, n)=\gamma(m, m-k, n) .
$$

This easy consequence of Proposition 1 appears to be not at all combinatorially obvious. In Section 6 we give a bijection which explains $(2.3)$ combinatorially.

Example 2. Let $m=5$ and $k=4$. There are seven $(5,4)$-capsid partitions of 16 :

$$
\left(1^{16}\right), \quad\left(4^{4}\right), \quad\left(1^{11}, 5\right), \quad\left(1^{6}, 5^{2}\right), \quad\left(1,5^{3}\right), \quad\left(1^{6}, 10\right), \quad\left(1^{2}, 14\right),
$$

so that $a(5,4,16)=7$, thus (see Example 1 confirming $a(5,4,16)=a(5,1,16)$. 
Apart from the concrete applications of $(m, k)$-capsids in this paper, we believe that this new class of partitions is an object of interest worthy of independent study from various (including combinatorial and analytic) points of view. In particular, it would be interesting to establish asymptotic formulas for the number of $(m, k)$-capsid partitions of $n$, as $n$ goes to infinity. For $t$-core partitions, this has been established by Anderson [1].

\section{Special $q$-Series identities involving CONGRUences}

The two Rogers-Ramanujan functions

$$
G(q):=\sum_{k=0}^{\infty} \frac{q^{k^{2}}}{(q ; q)_{k}} \quad \text { and } \quad H(q):=\sum_{k=0}^{\infty} \frac{q^{k(k+1)}}{(q ; q)_{k}}
$$

satisfy a number of remarkable properties. First of all, they factorize in closed form,

$$
G(q)=\frac{1}{\left(q, q^{4} ; q^{5}\right)_{\infty}} \quad \text { and } \quad H(q)=\frac{1}{\left(q^{2}, q^{3} ; q^{5}\right)_{\infty}}
$$

which are the two Rogers-Ramanujan identities [28].

We will make essential use of the beautiful identity

$$
1=H(q) G\left(q^{11}\right)-q^{2} G(q) H\left(q^{11}\right),
$$

which Ramanujan stated without proof in one of his letters to Hardy [25]. The first published proof is due to Rogers [29]. Ramanujan also gave another remarkable identity related to 3.1 :

$$
1=H(q) G(q)^{11}-q^{2} G(q) H(q)^{11}-11 q G(q)^{6} H(q)^{6} .
$$

Ramanujan's pair of equations (3.1) and (3.2) can be compared with the similar pair of identities

$$
1=\tilde{H}(q) \widetilde{G}\left(q^{3}\right)-q^{2} \tilde{G}(q) \tilde{H}\left(q^{3}\right)
$$

and

$$
1=\tilde{H}(q) \tilde{G}(q)^{3}-q^{2} \tilde{G}(q) \tilde{H}(q)^{3}-3 q \tilde{G}(q)^{2} \tilde{H}(q)^{2}
$$

where

$$
\tilde{G}(q)=\frac{1}{\left(q, q^{3}, q^{4}, q^{9}, q^{10}, q^{12} ; q^{13}\right)_{\infty}}
$$

and

$$
\widetilde{H}(q)=\frac{1}{\left(q^{2}, q^{5}, q^{6}, q^{7}, q^{8}, q^{11} ; q^{13}\right)_{\infty}},
$$

proved in S. Robins' 1991 thesis [27].

We define

$$
P_{m, k}(q):=\left(q^{k} ; q^{m}\right)_{\infty}\left(q^{m-k} ; q^{m}\right)_{\infty} .
$$

On one hand the symmetry

$$
P_{m, k}(q)=P_{m, m-k}(q)
$$


and the scaling of parameters

$$
P_{s m, s k}(q)=P_{m, k}\left(q^{s}\right)
$$

are obvious. On the other hand, using $(a ; q)_{\infty}=\left(a, a q ; q^{2}\right)_{\infty}$ and $(3.5)$, we also have

$$
P_{m, k}(q)=P_{2 m, k}(q) P_{2 m, m-k}(q) .
$$

Ramanujan's identity (3.1) can be written as

$$
1=\frac{1}{P_{5,2}(q) P_{55,11}(q)}-\frac{q^{2}}{P_{5,1}(q) P_{55,22}(q)},
$$

or, in view of 3.6 , as

$$
1=\frac{1}{P_{10,2}(q) P_{10,3}(q) P_{110,11}(q) P_{110,44}(q)}-\frac{q^{2}}{P_{10,1}(q) P_{10,4}(q) P_{110,22}(q) P_{110,33}(q)}
$$

which we will make use of.

Bressoud [5] gave an immediate combinatorial interpretation of (3.7), viewed as a mod 55 identity. From a combinatorial perspective, (3.7) is a "shifted partition identity". Other such identities (such as (3.3)), possibly related to other moduli, have been discussed and established in [3, 18, 11, 13]. Most of the known shifted partition identities share the feature that they can be written as some mod $m$ identity in the form

$$
1=A_{m}(q)-q^{d} B_{m}(q)
$$

with

$$
A_{m}(q)=\frac{1}{\prod_{j=1}^{12} P_{m, k_{j}}(q)} \quad \text { and } \quad B_{m}(q)=\frac{1}{\prod_{j=1}^{12} P_{m, l_{j}}(q)},
$$

for some $m, d \in \mathbb{N}$ and $0<k_{j}, l_{j}<m$, for $j=1, \ldots, 12$.

It is clear that (3.7) has the form of (3.9), obtained by taking $m=55, d=2, k_{j}=5 j-3$ and $l_{j}=5 j-4$ for $j=1 \ldots, 11, k_{12}=11$, and $l_{12}=22$. Similarly, (3.3) is a special case of (3.9), obtained by taking $m=39, d=2, k_{j}=13 j-11$ and $l_{j}=13 j-12$ for $j=1,2,3$, $k_{j}=13 j-47$ and $l_{j}=13 j-49$ for $j=4,5,6, k_{j}=13 j-85$ and $l_{j}=13 j-87$ for $j=7,8,9, k_{10}=3, k_{11}=9, k_{12}=12, l_{10}=6, l_{11}=15$, and $l_{12}=18$.

\section{Combinatorial interpretations of the tau FunCtion}

We are ready to collect the ingredients we prepared and apply them to obtain combinatorial interpretions of Ramanujan's tau function.

First observe that by Proposition 1 and the notation (3.4), the generating function for $(m, k)$-capsid partitions takes the form

$$
C_{m, k}(q)=\frac{\left(q^{m} ; q^{m}\right)_{\infty}}{P_{m, k}(q)}
$$


Now, we multiply both sides of $(3.8)$ by $q^{110}\left(q^{110} ; q^{110}\right)_{\infty}^{24}$ and rewrite the resulting identity in terms of $(10, k)$-capsid and 11-core generating functions:

$$
\begin{aligned}
\sum_{n=1}^{\infty} \tau(n) q^{110 n}= & q^{110} \frac{\left(q^{10} ; q^{10}\right)_{\infty}^{2}}{P_{10,2}(q) P_{10,3}(q)} \frac{\left(q^{110} ; q^{110}\right)_{\infty}^{2}}{P_{10,1}\left(q^{11}\right) P_{10,4}\left(q^{11}\right)} \frac{\left(q^{110} ; q^{110}\right)_{\infty}^{22}}{\left(q^{10} ; q^{10}\right)_{\infty}^{2}} \\
& -q^{112} \frac{\left(q^{10} ; q^{10}\right)_{\infty}^{2}}{P_{10,1}(q) P_{10,4}(q)} \frac{\left(q^{110} ; q^{110}\right)_{\infty}^{2}}{P_{10,2}\left(q^{11}\right) P_{10,3}\left(q^{11}\right)} \frac{\left(q^{110} ; q^{110}\right)_{\infty}^{22}}{\left(q^{10} ; q^{10}\right)_{\infty}^{2}} \\
= & q^{110} C_{10,2}(q) C_{10,3}(q) C_{10,1}\left(q^{11}\right) C_{10,4}\left(q^{11}\right) T_{11}\left(q^{10}\right)^{2} \\
& -q^{112} C_{10,1}(q) C_{10,4}(q) C_{10,2}\left(q^{11}\right) C_{10,3}\left(q^{11}\right) T_{11}\left(q^{10}\right)^{2}
\end{aligned}
$$

We let $\mathcal{C}_{m, k}$ be the set of $(m, k)$-capsids and $\mathcal{T}_{t}$ be the set of $t$-cores. We define two sets of vector partitions:

$$
\begin{aligned}
& \mathcal{A}:=\mathcal{C}_{10,2} \times \mathcal{C}_{10,3} \times \mathcal{C}_{10,1} \times \mathcal{C}_{10,4} \times \mathcal{T}_{11} \times \mathcal{T}_{11}, \\
& \mathcal{B}:=\mathcal{C}_{10,1} \times \mathcal{C}_{10,4} \times \mathcal{C}_{10,2} \times \mathcal{C}_{10,3} \times \mathcal{T}_{11} \times \mathcal{T}_{11}
\end{aligned}
$$

For a partition $\pi$ we let $|\pi|$ denote the sum of parts. For $\vec{\pi}=\left(\pi_{1}, \pi_{2}, \pi_{3}, \pi_{4}, \pi_{5}, \pi_{6}\right)$ in $\mathcal{A}$ or in $\mathcal{B}$ we define

$$
|\vec{\pi}|:=\left|\pi_{1}\right|+\left|\pi_{2}\right|+11 \cdot\left|\pi_{3}\right|+11 \cdot\left|\pi_{4}\right|+10 \cdot\left|\pi_{5}\right|+10 \cdot\left|\pi_{6}\right| \cdot
$$

If $|\vec{\pi}|=n$ we say that $\vec{\pi}$ is a vector partition of $n$. We let

$$
a(n):=\text { the number of vector partitions in } \mathcal{A} \text { of } n,
$$

and

$$
b(n):=\text { the number of vector partitions in } \mathcal{B} \text { of } n .
$$

Then it is clear that

$$
1+\sum_{n=1}^{\infty} a(n) q^{n}=C_{10,2}(q) C_{10,3}(q) C_{10,1}\left(q^{11}\right) C_{10,4}\left(q^{11}\right) T_{11}\left(q^{10}\right)^{2}
$$

and

$$
1+\sum_{n=1}^{\infty} b(n) q^{n}=C_{10,1}(q) C_{10,4}(q) C_{10,2}\left(q^{11}\right) C_{10,3}\left(q^{11}\right) T_{11}\left(q^{10}\right)^{2}
$$

We define $a(0)=1, b(-2)=b(-1)=0$, and $b(0)=1$. From 4.1) we readily obtain the following result:

Theorem 1 (A combinatorial formula for Ramanujan's tau function). For $n \geq 1$, we have

(i) $\quad \tau(n)=a(110 n-110)-b(110 n-112)$,

and

$$
0=a(n)-b(n-2), \quad \text { if } n \not \equiv 0 \bmod 110 \text {. }
$$


This formula for $\tau(n)$ has the advantage that it is compact and combinatorial, but the numbers obtained when computing $a(m)$ and $b(m)$ get large.

Example 3.

$$
\tau(2)=a(110)-b(108)=174780-174804=-24
$$

Let us look for another formula for $\tau(n)$ using a smaller modulus than 110, say 10. For this we use Ramanujan's (3.2) which we rewrite in the form

$$
\begin{aligned}
1=\frac{1}{P_{10,2}(q) P_{10,3}(q) P_{10,1}(q)^{11} P_{10,4}(q)^{11}} & -\frac{q^{2}}{P_{10,1}(q) P_{10,4}(q) P_{10,2}(q)^{11} P_{10,3}(q)^{11}} \\
& -\frac{11 q}{P_{10,1}(q)^{6} P_{10,4}(q)^{6} P_{10,2}(q)^{6} P_{10,3}(q)^{6}} .
\end{aligned}
$$

We multiply both sides of this relation by $q^{10}\left(q^{10} ; q^{10}\right)_{\infty}^{24}$ and rewrite the resulting identity in terms of $(10, k)$-capsid generating functions:

$$
\begin{aligned}
\sum_{n=1}^{\infty} \tau(n) q^{10 n}= & q^{10} C_{10,2}(q) C_{10,3}(q) C_{10,1}(q)^{11} C_{10,4}(q)^{11} \\
& -q^{12} C_{10,1}(q) C_{10,4}(q) C_{10,2}(q)^{11} C_{10,3}(q)^{11} \\
& -11 q^{11} C_{10,1}(q)^{6} C_{10,4}(q)^{6} C_{10,2}(q)^{6} C_{10,3}(q)^{6}
\end{aligned}
$$

We now define three sets of vector partitions, each defined by a twentyfour-fold Cartesian product:

$$
\mathcal{U}:=X_{j=1}^{24} \mathcal{U}_{j}, \quad \mathcal{V}:=X_{j=1}^{24} \mathcal{V}_{j}, \quad \mathcal{W}:=X_{j=1}^{24} \mathcal{W}_{j}
$$

where

$$
\begin{aligned}
& \mathcal{U}_{1}=\mathcal{C}_{10,2}, \quad \mathcal{U}_{2}=\mathcal{C}_{10,3}, \quad \mathcal{U}_{j}=\mathcal{C}_{10,1}, \quad \mathcal{U}_{j+11}=\mathcal{C}_{10,4}, \\
& \mathcal{V}_{1}=\mathcal{C}_{10,1}, \quad \mathcal{V}_{2}=\mathcal{C}_{10,4}, \quad \mathcal{V}_{j}=\mathcal{C}_{10,2}, \quad \mathcal{V}_{j+11}=\mathcal{C}_{10,3}, \quad \text { for } j=3, \ldots, 13 \text {, } \\
& \mathcal{W}_{l}=\mathcal{C}_{10,1}, \quad \mathcal{W}_{l+6}=\mathcal{C}_{10,4}, \quad \mathcal{W}_{l+12}=\mathcal{C}_{10,2}, \quad \mathcal{W}_{l+18}=\mathcal{C}_{10,3}, \quad \text { for } l=1, \ldots, 6 \text {. }
\end{aligned}
$$

For $\vec{\pi}=\left(\pi_{1}, \pi_{2}, \ldots, \pi_{24}\right)$ in $\mathcal{U}, \mathcal{V}$, or in $\mathcal{W}$ we define

$$
|\vec{\pi}|:=\sum_{j=1}^{24}\left|\pi_{j}\right| .
$$

If $|\vec{\pi}|=n$ we say that $\vec{\pi}$ is a vector partition of $n$. We let

$$
\begin{aligned}
u(n) & :=\text { the number of vector partitions in } \mathcal{U} \text { of } n, \\
v(n) & :=\text { the number of vector partitions in } \mathcal{V} \text { of } n \\
w(n) & :=\text { the number of vector partitions in } \mathcal{W} \text { of } n
\end{aligned}
$$


Then it is clear that

$$
\begin{aligned}
& 1+\sum_{n=1}^{\infty} u(n) q^{n}=C_{10,2}(q) C_{10,3}(q) C_{10,1}(q)^{11} C_{10,4}(q)^{11}, \\
& 1+\sum_{n=1}^{\infty} v(n) q^{n}=C_{10,1}(q) C_{10,4}(q) C_{10,2}(q)^{11} C_{10,3}(q)^{11}, \\
& 1+\sum_{n=1}^{\infty} w(n) q^{n}=C_{10,1}(q)^{6} C_{10,4}(q)^{6} C_{10,2}(q)^{6} C_{10,3}(q)^{6} .
\end{aligned}
$$

We shall define $u(0)=1, v(-2)=v(-1)=0, v(0)=1, w(-1)=0$ and $w(0)=1$. From (4.2) we readily obtain the following result:

Theorem 2 (Another combinatorial formula for Ramanujan's tau function). For $n \geq 1$, we have

(i) $\quad \tau(n)=u(10 n-10)-v(10 n-12)-11 w(10 n-11)$,

and

$$
0=u(n)-v(n-2)-11 w(n-1), \quad \text { if } n \not \equiv 0 \bmod 10 .
$$

Computationally, this formula is not better than the one in Theorem 1 , as the numbers obtained when computing $u(l), v(l)$, and $w(l)$ get large as well. In particular (compare with Example 3p

$$
\tau(2)=u(10)-v(8)-11 w(9)=381405-3139-11 \cdot 34390=-24 .
$$

\section{OTHER COMBINATORIAL INTERPRETATIONS}

It is clear that one can also employ other shifted partition identities (which are listed in [13]) to obtain combinatorial interpretations of Ramanujan's tau function which are similar to those in Theorems 1 and 2. Also, one can in principle use those shifted partition identities to obtain combinatorial interpretations for powers other than 24 of Dedekind's eta function. On the contrary, our method can also be used to establish that certain sets of Cartesian products involving (restricted) partitions, $(m, k)$-capsid partitions and $t$-core partitions have the same cardinality.

For example, Jacobi showed that

$$
(q ; q)_{\infty}^{3}=\sum_{n=0}^{\infty}(-1)^{n}(2 n+1) q^{13\left(\begin{array}{c}
n+1 \\
2
\end{array}\right)} .
$$

Joichi and Stanton [17] have found a combinatorial proof of (5.1). Combining the $q \mapsto q^{13}$ case of this identity with (3.3), we have

$$
\sum_{n=0}^{\infty}(-1)^{n}(2 n+1) q^{13\left(\begin{array}{c}
n+1 \\
2
\end{array}\right)}=\frac{C_{13,2}(q) C_{13,5}(q) C_{13,6}(q)}{P_{13,1}\left(q^{3}\right) P_{13,3}\left(q^{3}\right) P_{13,4}\left(q^{3}\right)}-q^{2} \frac{C_{13,1}(q) C_{13,3}(q) C_{13,4}(q)}{P_{13,2}\left(q^{3}\right) P_{13,5}\left(q^{3}\right) P_{13,6}\left(q^{3}\right)},
$$


which leads to the following combinatorial interpretation:

Let $\mathcal{D}$ and $\mathcal{E}$ be two sets of vector partitions, defined by

$$
\begin{aligned}
\mathcal{D} & :=\mathcal{C}_{13,2} \times \mathcal{C}_{13,5} \times \mathcal{C}_{13,6} \times X, \\
\mathcal{E} & :=\mathcal{C}_{13,1} \times \mathcal{C}_{13,3} \times \mathcal{C}_{13,4} \times Y,
\end{aligned}
$$

where $X$ is the set of partitions with parts $\pm 1, \pm 3, \pm 4 \bmod 13$, and $Y$ is the set of partitions with parts $\pm 2, \pm 5, \pm 6 \bmod 13$. For $\vec{\pi}=\left(\pi_{1}, \pi_{2}, \pi_{3}, \pi_{4}\right)$ in $\mathcal{D}$ or in $\mathcal{E}$ we define

$$
|\vec{\pi}|:=\left|\pi_{1}\right|+\left|\pi_{2}\right|+\left|\pi_{3}\right|+3 \cdot\left|\pi_{4}\right| \cdot
$$

If $|\vec{\pi}|=n$ we say that $\vec{\pi}$ is a vector partition of $n$. We let

$$
d(n):=\text { the number of vector partitions in } \mathcal{D} \text { of } n,
$$

and

$$
e(n):=\text { the number of vector partitions in } \mathcal{E} \text { of } n .
$$

Then, with $e(-1)=0$ and $e(0)=1$, we have the following result involving triangular numbers:

Theorem 3. For $m \geq 1$, we have

$$
\text { (i) } \quad d(m)-e(m-2)=(-1)^{n}(2 n+1), \quad \text { if } m=\frac{13 n(n+1)}{2}
$$

and

$$
\text { (ii) } \quad d(m)-e(m-2)=0, \quad \text { otherwise. }
$$

\section{CAPSID SYMMetries}

In this section we explain (2.3) combinatorially. In Section 2 we defined $(m, k)$-capsids. Now we define more general capsids. Suppose $m, r_{1}, r_{2} \in \mathbb{N}, m \geq 2$ and $0<r_{1} \neq r_{2}<m$. We say that a partition $\pi$ is an $\left(m, r_{1}, r_{2}\right)$-capsid if, and only if, the possible parts of $\pi$ are $r_{1}$ or are congruent to 0 or $r_{2} \bmod m$, and satisfy the following two conditions:

(i) if $\mu_{r_{1}}=0$, i.e., $r_{1}$ is not a part, then all parts are congruent to $r_{2} \bmod m$;

(ii) If $\mu_{r_{1}}>0$, then $r_{1}$ is the smallest part and the largest part congruent to $0 \bmod m$ is $\leq m \cdot \mu_{r_{1}}$ and all parts congruent to $r_{2} \bmod m$ are $>m \cdot \mu_{r_{1}}$.

Thus (for $k \neq m / 2)$ a $(m, k)$-capsid is a $(m, m-k, k)$-capsid.

Let $\gamma\left(m, r_{1}, r_{2}, n\right)$ be the number of $\left(m, r_{1}, r_{2}\right)$-capsid partitions of $n$. We have the following generating function

$$
C_{m, r_{1}, r_{2}}(q)=1+\sum_{n=1}^{\infty} \gamma\left(m, r_{1}, r_{2}, n\right) q^{n}=\sum_{n=0}^{\infty} \frac{q^{r_{1} n}}{\left(q^{m} ; q^{m}\right)_{n}\left(q^{m n+r_{2}} ; q^{m}\right)_{\infty}}
$$

For an $\left(m, r_{1}, r_{2}\right)$-capsid $\pi$ we define two statistics:

(i) We let $\alpha(\pi)=\mu_{r_{1}}$.

(ii) We let $\beta(\pi)$ be the number of parts of $\pi$ that are congruent to $r_{2} \bmod m$. 
Let $\gamma\left(m, r_{1}, r_{2}, a, b, n\right)$ be the number of $\left(m, r_{1}, r_{2}\right)$-capsid partitions $\lambda$ of $n$ with $\alpha(\lambda)=$ $a$ and $\beta(\lambda)=b$.

Let $\mathcal{C}_{m, r_{1}, r_{2}}$ be the set of $\left(m, r_{1}, r_{2}\right)$-capsids. It is clear that we have the following generating function

$$
C_{m, r_{1}, r_{2}}(x, y, q)=\sum_{\lambda \in \mathcal{C}_{m, r_{1}, r_{2}}} x^{\alpha(\lambda)} y^{\beta(\lambda)} q^{|\lambda|}=\sum_{n=0}^{\infty} \frac{x^{n} q^{r_{1} n}}{\left(q^{m} ; q^{m}\right)_{n}\left(y q^{m n+r_{2}} ; q^{m}\right)_{\infty}} .
$$

By (2.2) we have the following result:

Proposition 2. The generating function for $\left(m, r_{1}, r_{2}\right)$-capsids is

$$
C_{m, r_{1}, r_{2}}(x, y, q)=\frac{\left(x y q^{r_{1}+r_{2}} ; q^{m}\right)_{\infty}}{\left(x q^{r_{1}} ; q^{m}\right)_{\infty}\left(y q^{r_{2}} ; q^{m}\right)_{\infty}} .
$$

Since $C_{m, r_{1}, r_{2}}(x, y, q)=C_{m, r_{2}, r_{1}}(y, x, q)$ and

$$
\gamma\left(m, r_{1}, r_{2}, a, b, n\right)=\text { the coefficient of } x^{a} y^{b} q^{n} \text { in } C_{m, r_{1}, r_{2}}(x, y, q),
$$

we deduce that

$$
\gamma\left(m, r_{1}, r_{2}, a, b, n\right)=\gamma\left(m, r_{2}, r_{1}, b, a, n\right)
$$

for all $n$. By summing over $a, b$ this implies that

$$
\gamma\left(m, r_{1}, r_{2}, n\right)=\gamma\left(m, r_{2}, r_{1}, n\right)
$$

for all $n$, which generalizes $(2.3)$.

In this section we give a combinatorial proof of (6.3) by constructing a bijection

$$
\mathcal{J}: \mathcal{C}\left(m, r_{1}, r_{2}, a, b, n\right) \longrightarrow \mathcal{C}\left(m, r_{2}, r_{1}, b, a, n\right),
$$

where

$$
\mathcal{C}\left(m, r_{1}, r_{2}, a, b, n\right):=\left\{\lambda \in \mathcal{C}_{m, r_{1}, r_{2}}: \alpha(\lambda)=a, \beta(\lambda)=b,|\lambda|=n\right\} .
$$

For a given $\lambda \in \mathcal{C}\left(m, r_{1}, r_{2}, a, b, n\right)$ we divide each part of $\lambda$ congruent to 0 mod $m$ by $m$ to form a partition $\pi_{1}$, and form a partition $\pi_{2}$ by subtracting $r_{2}$ from each part of $\lambda$ congruent to $r_{2} \bmod m$ and dividing by $m$. Then we form the diagram given below in Figure 1.

\section{Note:}

(i) The partition $\pi_{2}$ has $b$ parts each $\geq a$.

(ii) The parts of $\pi_{1}$ are $\leq a$.

(iii) $a$ is the number of smallest parts of $\lambda$.

(iv) If $b=0$ then $\pi_{2}=()$ is empty.

(v) It is possible that $\pi_{1}=()$ is empty.

Next we form the partition

$$
\pi=\pi_{2} \cup \pi_{1}
$$

and take the conjugate $\pi^{\prime}$. Then we form partitions $\tilde{\pi}_{1}, \tilde{\pi}_{2}$, where $\pi^{\prime}=\tilde{\pi}_{2} \cup \tilde{\pi}_{1}$ and given in the diagram below in Figure 2. 


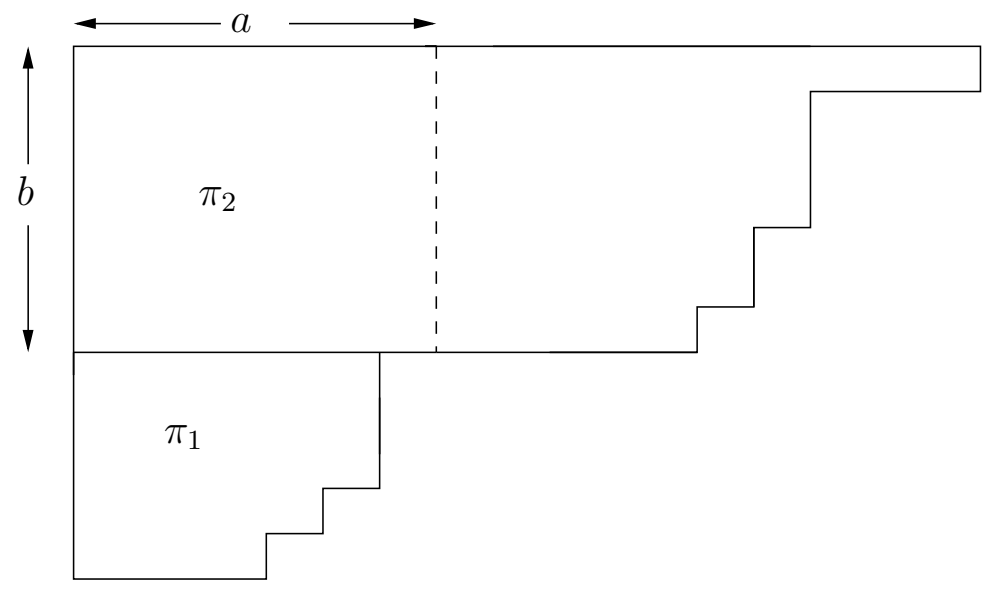

FIGURE 1. The partition $\pi=\pi_{2} \cup \pi_{1}$

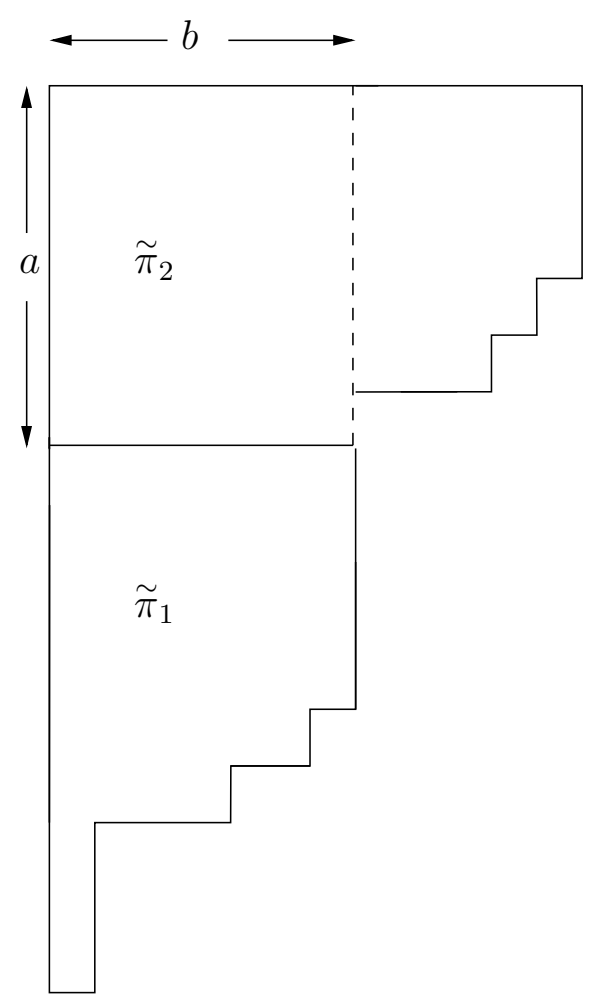

FiguRE 2. The partition $\pi^{\prime}=\tilde{\pi}_{2} \cup \tilde{\pi}_{1}$

We form the partition $\tilde{\lambda}$ with $b$ smallest parts $r_{2}$, with parts from $\tilde{\pi}_{1}$ multiplied by $m$, and with adding $r_{1}$ to each part of $\tilde{\pi}_{2}$ after multiplying by $m$. We see that $\tilde{\lambda} \in$ $\mathcal{C}\left(m, r_{2}, r_{1}, b, a, n\right)$. We define $\mathcal{J}(\lambda)=\tilde{\lambda}$ and $\mathcal{J}$ is a bijection. 
Example 4. $\lambda=\left(1^{3}, 5^{1}, 15^{2}, 22^{1}, 27\right), m=5, r_{1}=1, r_{2}=2, a=3, b=2$ and $n=87$. From Figure 3 below we see that $\mathcal{J}(\lambda)=\tilde{\lambda}=\left(2^{2}, 5^{1}, 10^{1}, 21^{2}, 26\right) \in \mathcal{C}(5,2,1,2,3,87)$.
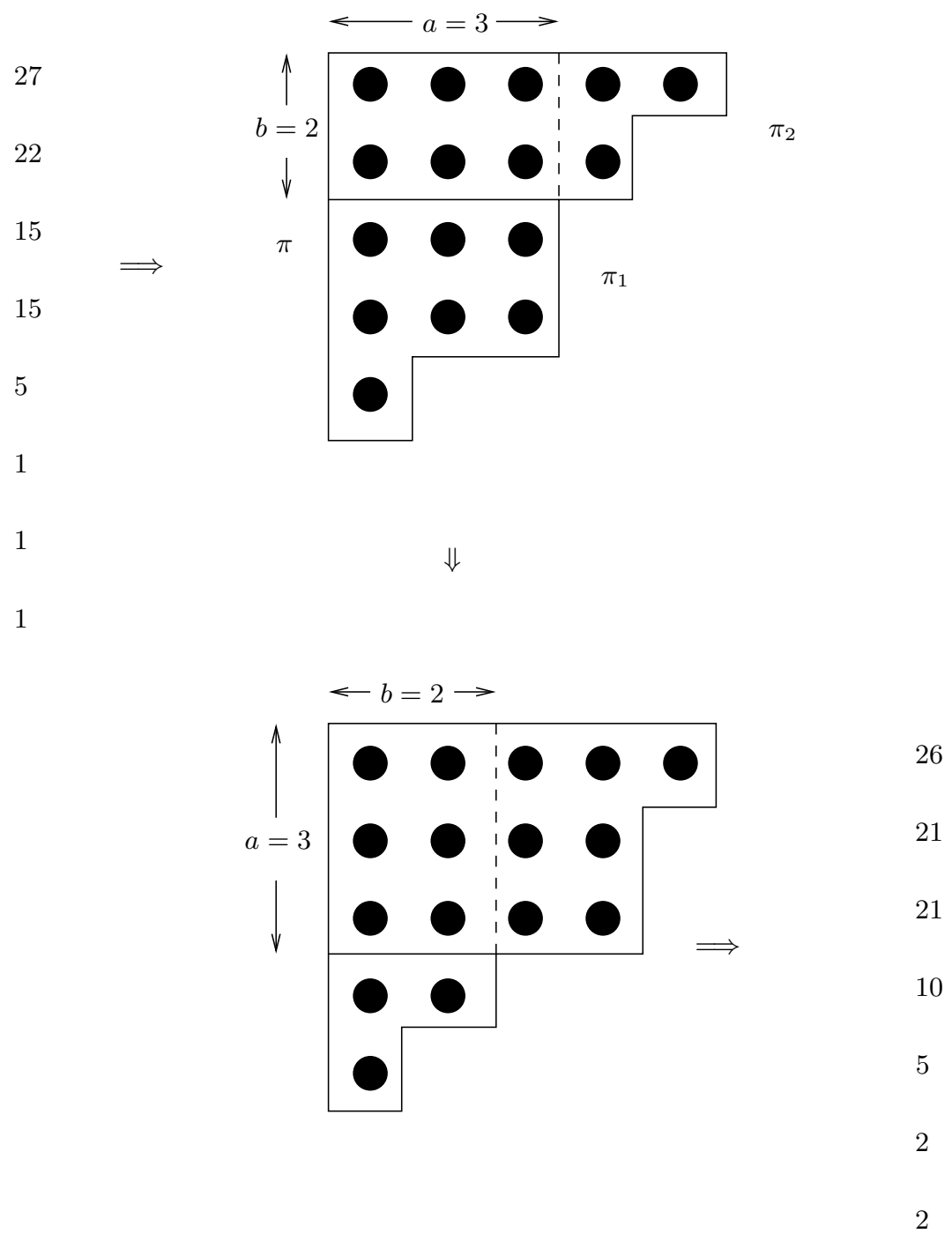

FigurE 3. Example of bijection $\mathcal{J}$

\section{Concluding Remarks}

The main goal of this paper was to give a combinatorial interpretation of the Ramanujan tau function $\tau(n)$ in terms of partitions. In Theorems 1 and 2 we were able to write $\tau(n)$ as the difference of two, resp. three partition functions with nonnegative coefficients. We do not claim that this should be used to calculate $\tau(n)$ or to find improved asymptotics. 
Charles [6] and Edixhoven [8] have found efficient algorithms for computing $\tau(n)$. We note that there are other ways to express $\tau(n)$ in terms of nonnegative arithmetic functions. For example it is well known that there are many identities for $\tau(n)$ in terms of divisor functions. Following Ramanujan [24, p.163] we define

$$
\Phi_{j}(q)=\sum_{n=1}^{\infty} \frac{n^{j} q^{n}}{1-q^{n}}=\sum_{m, n \geq 1} n^{j} q^{n m}=\sum_{n=1}^{\infty} \sigma_{j}(n) q^{n},
$$

for $j \geq 1$ odd and where $\sigma_{j}(n)=\sum_{d \mid n} d^{j}$. For $n \geq 2$ even we define the Eisenstein series

$$
E_{n}(q)=1-\frac{2 n}{B_{n}} \Phi_{n-1}(q),
$$

where $B_{n}$ is the $n$-th Bernoulli number. Then it is well-known that

$$
\sum_{n=1}^{\infty} \tau(n) q^{n}=\frac{1}{1728}\left(E_{4}(q)^{3}-E_{6}(q)^{2}\right) .
$$

See [21, p.172] for a history of this identity. Thus $\tau(n)$ can be written in terms of the divisor sums $\sigma_{3}(n)$ and $\sigma_{5}(n)$. A nicer identity is

$$
\sum_{n=1}^{\infty} \tau(n) q^{n}=\frac{691}{762048}\left(E_{12}(q)-E_{6}(q)^{2}\right)
$$

which implies that

$$
\tau(n)=\frac{65}{756} \sigma_{11}(n)+\frac{691}{756} \sigma_{5}(n)-\frac{691}{3} \sum_{m=1}^{n-1} \sigma_{5}(m) \sigma_{5}(n-m) .
$$

See [22, Eq.(1.15)]. It is not clear how to interpret the right side of $(7.2)$ combinatorially, although it does express $\tau(n)$ in terms of nonnegative arithmetic functions.

\section{REFERENCES}

[1] J. Anderson, "An asymptotic formula for the $t$-core partition function and a conjecture of Stanton". J. Number Theory (9) 128 (2008), 2591-2615.

[2] G. E. Andrews, The theory of partitions, Cambridge Univ. Press, Cambridge, 1976.

[3] G. E. Andrews, "Further problems on partitions", Amer. Math. Monthly (5) 94 (1987), 437-439.

[4] A. Berkovich and F. G. Garvan, "K. Saito's conjecture for nonnegative eta products and analogous results for other infinite products", J. Number Theory 128 (2008), 1731-1748.

[5] D. M. Bressoud, Proof and generalization of certain identities conjectured by Ramanujan, Ph.D. thesis, Temple University, 1977, $187 \mathrm{pp}$.

[6] D. X. Charles, "Computing the Ramanujan tau function", Ramanujan J. 11 (2006), 221-224.

[7] F. J. Dyson, "Missed opportunities", Bull. Amer. Math. Soc. (5) 78 (1972), 635-652.

[8] B. Edixhoven, "On the computation of the coefficients of a modular form", in Algorithmic number theory, Lecture Notes in Comput. Sci., Vol. 4076, Springer, Berlin, 2006, pp. 30-39.

[9] F. G. Garvan, "Combinatorial interpretations of Ramanujan's partition congruences", in Ramanujan Revisited: Proc. of the Centenary Conference, Univ. of Illinois at Urbana-Champaign, June 1-5, 1987, pp. 29-45, Academic Press, Boston, MA, 1988. 
[10] F. G. Garvan, "Some congruences for partitions that are t-cores", Proc. London Math. Soc. 66 (1993), 449-478.

[11] F. G. Garvan, "Shifted and shiftless partition identities", in Number theory for the millennium, II (Urbana, IL, 2000), A.K. Peters, Natick, MA, 2002; pp. 75-92.

[12] F. G. Garvan, D. Kim and D. Stanton, "Cranks and t-cores", Invent. Math. (1) 101 (1990), 1-17.

[13] F. G. Garvan and H. Yesilyurt, "Shifted and shiftless partition identities. II", Int. J. Number Th. (1) 3 (2007), 43-84.

[14] G. Gasper and M. Rahman, Basic hypergeometric series, second edition, Encyclopedia of Mathematics and Its Applications 96, Cambridge University Press, Cambridge, 2004.

[15] A. Granville and K. Ono, "Defect zero p-blocks for finite simple groups", Trans. Amer. Math. Soc. (1) 348 (1996), 331-347.

[16] G. James and A. Kerber, The representation theory of the symmetric group, Addison-Wesley, Reading, 1981.

[17] J. T. Joichi and D. Stanton, "An involution for Jacobi's identity", Discrete Math. 73 (1989), 261-271.

[18] A. Kalvade, "Equality of shifted partition functions", J. Indian Math. Soc. (N.S.) (1-4) 54 (1989),155-164.

[19] D. H. Lehmer, "The vanishing of Ramanujans function $\tau(n)$ ", Duke Math. J. 14 (1947), 429-433.

[20] I. G. Macdonald, "Affine root systems and Dedekinds $\eta$-function", Invent. Math. 15 (1972), 91-143.

[21] S. C. Milne, "Hankel determinants of Eisenstein series", in Symbolic computation, number theory, special functions, physics and combinatorics (Gainesville, FL, 1999), Dev. Math., Vol. 4, Kluwer Acad. Publ., Dordrecht, 2001, pp. 171-188.

[22] S. C. Milne, "Infinite families of exact sums of squares formulas, Jacobi elliptic functions, continued fractions, and Schur functions", Ramanujan J. 6 (2002), 7-149.

[23] L. J. Mordell, "On Mr. Ramanujan's empirical expansions of modular functions", Proc. Cambridge Philos. Soc. 19 (1917), 117-124.

[24] S. Ramanujan, "On certain arithmetical functions", Trans. Cambridge Philos. Soc. (9) 22 (1916), 159-184.

[25] S. Ramanujan, "Algebraic relations between certain infinite products", Proc. London Math. Soc. (2) 18 (1920), p. xviii.

[26] R. A. Rankin, "Ramanujan's tau-function and its generalizations", in Ramanujan revisited (G.E. Andrews, ed.; Urbana-Champaign, IL, 1987), Boston, MA: Academic Press, pp. 245-268, 1988.

[27] S. Robins, Arithmetic properties of modular forms, $\mathrm{PhD}$ thesis, University of California, Los Angeles, 1991.

[28] L. J. Rogers, "Second memoir on the expansion of certain infinite products", Proc. London Math. Soc. 25 (1894), 318-343.

[29] L. J. Rogers, "On a type of modular relation", Proc. London Math. Soc. 19 (1921), 387-397.

Department of Mathematics, University of Florida, 358 Little Hall, PO box 118105, Gainesville, FL 32611-8105, USA

E-mail address: fgarvan@ufl.edu

URL: http://www.qseries.org/fgarvan/

Fakultät für Mathematik, Universität Wien, Oskar-Morgenstern-Platz 1, A-1090 ViENNA, AUSTRIA

E-mail address: michael.schlosser@univie.ac.at

$U R L:$ http://www.mat.univie.ac.at/〜schlosse 